\title{
Fine and Wide Frequency Tuning Digital Controlled Oscillators Utilizing Capacitance Position Sensitivity in Distributed Resonators
}

\author{
Win Chaivipas, Takeshi Ito, Takashi Kurashina, Kenichi Okada, and Akira Matsuzawa, \\ Department of Physical Electronics \\ Tokyo Institute of Technology \\ 2-12-1-S3-27 Ookayama, Meguro-ku, Tokyo 152-8552 Japan \\ win_chaivipas@ssc.pe.titech.ac.jp
}

\begin{abstract}
Recent advances have shown that all digital phase locked loops have several desirable advantages over its analog counterpart. One important element in the all digital phase locked loop is the digital controlled oscillator whose frequency resolution restricts the frequency resolution of the entire system. In this paper we propose a new structure for digital controlled oscillators utilizing the capacitance's sensitivity dependence on position of the shorted transmission line to increase the frequency resolution. A 9GHz transmission line based digital controlled oscillator was fabricated as a proof of concept. Measured results show that more than 100 times frequency step resolution increase is possible utilizing the same tuning capacitor size located at different points in the transmission line.
\end{abstract}

\section{INTRODUCTION}

Interest in time domain signal processing of signals at RF frequencies have spurred an increased interest in circuits such as all-digital phase locked loops. Aside from its advantages of having less process, voltage and temperature (PVT) dependence it also allows such techniques as direct frequency modulation $[1,2]$, and allows ease of implementation of fast settling techniques such as dynamic filter bandwidth control [3], and direct reference feed-forwarding method $[4,5]$.

In all-digital phase locked loop frequency synthesizers, one of the design challenges is the design of a digital controlled oscillator (DCO) with enough frequency controlling resolution for accurate frequency synthesis, and wide enough tuning range for PVT calibration. For example [6] utilizes 7-bit binary resolution for PVT calibration, 64-bit unit for acquisition, 128bit unit for tracking, and 3 bit unit for fractional tracking. This leads to more than 20bits tunable range. Such high dynamic range requires capacitor of several orders of difference in size, and the resolution limit of the DCO is fundamentally governed by the size of the capacitor that can be created. Working with such wide range of capacitance sizes requires switching very small capacitances, for example [7] mentions utilizing MOS varactors with as small as $50 \mathrm{aF}$ in $90 \mathrm{~nm}$ CMOS technology. Switching such small capacitors will be difficult as switch and varactor biasing parasitic capacitance can easily exceed the size of the capacitor. In addition, these parasitic problems will become more significant at higher frequencies. While using signal processing techniques to increase the effective resolution such as high speed delta sigma dithering is possible; for example [1] mentions 8 bit were dithered at $600 \mathrm{MHz}$ to increase the effective resolution, this is at the cost of significant power and it would be desirable if these requirements could be decreased.

In this paper we introduce a new DCO which helps to alleviate the issue of controlling very small capacitance. By utilizing the characteristic of the capacitor's sensitivity with relation to its position on a shorted transmission line simultaneous wide frequency tuning range and very fine frequency tuning steps is achieved.

\section{SHORTED TRANSMISSION LINE RESONATOR ANALYSIS}

It is well known that transmission lines can be utilized as resonators. In particular, shorted transmission lines are popular resonators as they exhibit anti-resonance which is the same oscillation mode to the parallel LC tank commonly used in differential oscillators. A typical transmission line resonator based oscillator is shown in Fig. 1. It is generally known that the shorted transmission line will resonate in anti-resonance when its electrical length is $\mathrm{N} 4$. The input impedance looking into a shorted transmission line length $l$, as in Fig. 2, and with propagation constant $\beta$ is (1).

$$
Z_{\text {in }}=j \cdot Z_{0} \cdot \tan (\beta \cdot l)=j \cdot Z_{0} \cdot \frac{\sin (\beta \cdot l)}{\cos (\beta \cdot l)}
$$
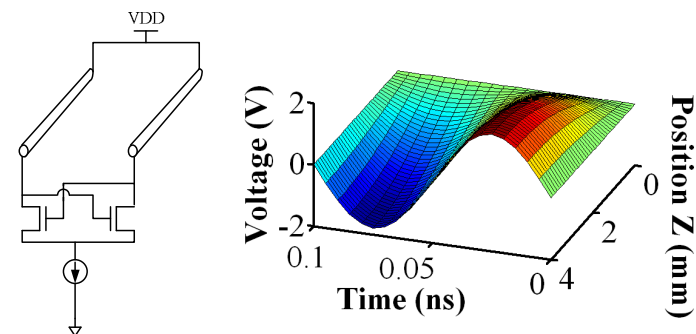

Fig. 1. Typical Transmission line based resonator and voltage wave. 


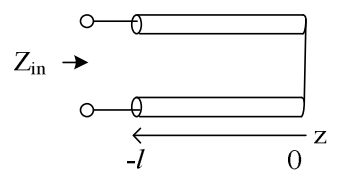

(a)

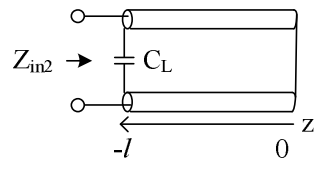

(b)
Fig. 2. Shorted transmission line (a), and shorted transmission line with input capacitor (b).

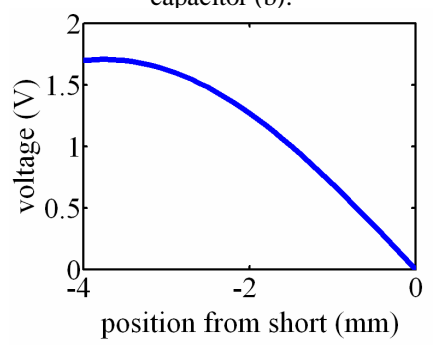

Fig. 3. Voltage amplitude of shorted transmission line.

Anti-resonance occurs when the impedance is maximum, which occurs when the denominator is zero, making the impedance go to infinity.

$$
\cos (\beta \cdot l)=0 .
$$

Then the resonance frequency can be found as

$$
f_{0}=\frac{v_{p}}{4 \cdot l}=\frac{1}{4 \cdot l \cdot \sqrt{\mu_{0} \cdot \varepsilon_{0} \cdot \varepsilon_{\text {reff }}}} .
$$

Where $\mathrm{v}_{\mathrm{p}}$ and $\varepsilon_{\text {reff }}$ are the phase velocity and the effective relative dielectric constant respectively. In general, the voltage as a function of position on a shorted transmission line can be expressed as

$$
V(z)=V_{0}^{+} \cdot \Re\left(e^{j \cdot(\omega \cdot t-\beta \cdot z)}-e^{j \cdot(\omega \cdot t+\beta \cdot z)}\right) .
$$

Where $\mathfrak{R}(\mathrm{f})$ represents the real part of ' $f$ ', and $V_{0}^{+}$represents the forward propagating voltage wave component. The waveform of a section of an approximately $\lambda / 4$ shorted transmission line at $10 \mathrm{GHz}$ at one time instant is shown in Fig. 3. The current waveform is the opposite of the voltage waveform with the maximum current at the shorted end and zero current at $\lambda / 4$, phase shifted by $\pi / 2$ radians.

Usually a tuning capacitor or capacitor bank is placed as shown in Fig.2 (b). Following the same method earlier, (5) must be satisfied for resonance to occur.

$$
\cos (\beta \cdot l)-\omega \cdot C_{L} \cdot Z_{0} \cdot \sin (\beta \cdot l)=0
$$

It is difficult to find a simple expression for $\omega$ from (5), (note that $\beta=\omega / v_{p}$ ). The approximation (6) can simplify the equation to $(5)$, leading to $(7)$

$$
\omega \cdot C_{L} \cdot Z_{0}<<1
$$

Note that $Z_{0}=1 /\left(v_{p}{ }^{*} C^{\prime}\right)$ [8] where $C^{\prime}$ is the capacitance per unit length of the transmission line. Equ. (7) clearly shows how the

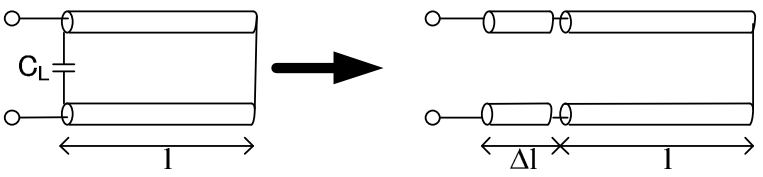

Fig. 4. In terms of resonance frequency the following approximation can be made.

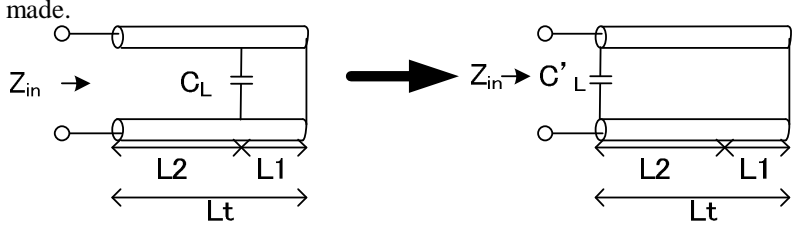

Fig. 5. Capacitance in the middle of the transmission line is reflected to an equivalent input capacitance.

capacitance $\mathrm{C}_{\mathrm{L}}$ affects the drop in resonance frequency. It should be noted that for a small

$$
f_{0} \approx \frac{1}{4 \cdot\left(\left(l+C_{L} / C^{\prime}\right) \cdot \sqrt{\mu_{0} \cdot \varepsilon_{0} \cdot \varepsilon_{\text {reff }}}\right)} .
$$

to medium value of $C_{L}$ and $\omega$ with respect to $\beta$ and 1 (7) holds well. For example simulation has shown that for $C_{L}=0.1 \mathrm{pF}$ at approximately $10 \mathrm{GHz}(7)$ is accurate to within $0.5 \%$ while for $C_{L}$ $=0.3 \mathrm{pF}$ it is accurate to within $6 \%$ in predicting the resonance frequency. For $10 \mathrm{GHz}$ DCOs these values are reasonably large, and so the accuracy of this equation should be enough to cover circuits with reasonable tuning range. From (7) it is interesting to note that in terms of the resonance frequency, the case with loaded capacitor can be approximated with an equivalent resonator without a capacitor but with a length extension of $\Delta l=C_{L} / C^{\prime}$ as shown in Fig.4. This interpretation is handy for a quick, intuitive estimation of how the resonance frequency will change with different size capacitors.

\section{Position Based CAPAcITANCE SEnSitivity}

From the voltage curve in Fig. 3 it is seen that the voltage amplitude is highest at the input and zero at the short circuit end. Since the capacitance is related to the voltage and current over it, it is reasonable to expect that the amount of voltage and current varying with the position of the transmission line would affect the effective input impedance contribution of the capacitance to the transmission line. This impedance contribution, in turn, is expected to contribute to the resonance frequency differently according to the capacitance position on the transmission line.

Approximations as to how much the capacitor contributes to the resonance frequency when placed at an arbitrary position on the transmission line are not straight forward. For example following the previous method for finding the resonance frequency and utilizing Taylor expansion estimation will lead to good prediction results, but the resulting equation is complex, difficult to interpret, and does not give an intuitive idea as to how the capacitance at various positions affects the resonance frequency. 


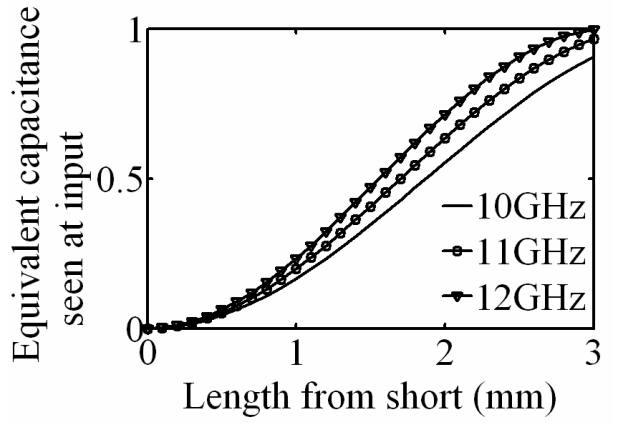

Fig. 6. Equivalent normalized reflected capacitance example.

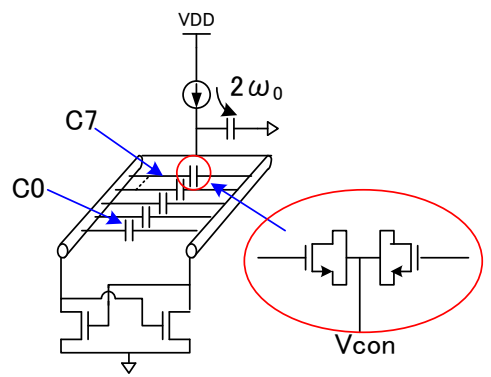

Fig. 7. Experimental prototype new DCO.

One method that can be utilized is using a capacitance transformation, i.e., by reflecting the capacitor from any point on the transmission line to the input as shown in Fig. 5. The equivalent input capacitance is found by equating the equations governing resonance condition of the circuits and is found to be

$$
\begin{aligned}
C_{L}{ }^{\prime}=\frac{C_{L}}{2} & +\left(\frac{C_{L}}{2}\right) \cdot \sin \left(\frac{\omega}{v_{p}} \cdot(2 \cdot L 1)\right) \cdot \cot \left(\frac{\omega}{v_{p}} \cdot L t\right) \\
& -\left(\frac{C_{L}}{2}\right) \cdot \cos \left(\frac{\omega}{v_{p}} \cdot(2 \cdot L 1)\right) .
\end{aligned}
$$

From (8) for example, it can be readily calculated that the effective capacitance located exactly in the middle of the transmission line is equal to only half its value if it were to be located at the input of the transmission line. Its effect on resonance frequency is then estimated iteratively by (7).

Fig.6 shows an example of how the equivalent effective input capacitance value changes with respect to position. It is seen that the effective value of the capacitance decreases as it approaches the shorted end which is attributed by the fact that as the capacitor's position comes nearer to the short, most of the current flows through the short making the capacitance's contribution seen at the input decrease.

\section{Using The CAPACITANCE Sensitivity In DCO}

Analysis from section III opens up a great potential for designing digital controlled oscillators. The resonance frequency can now be tuned by, not only scaling the capacitors size, but also by varying the capacitor's position along the shorted

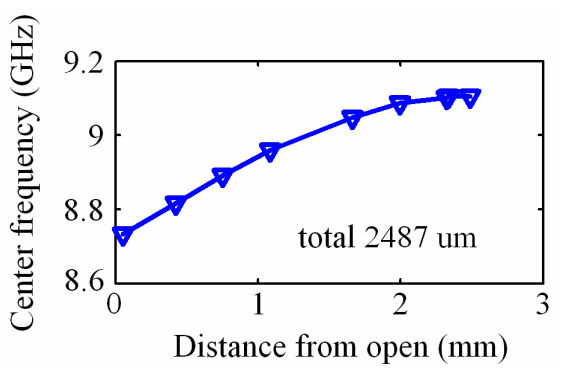

Fig. 8. Oscillation frequency vs switching position from open end.

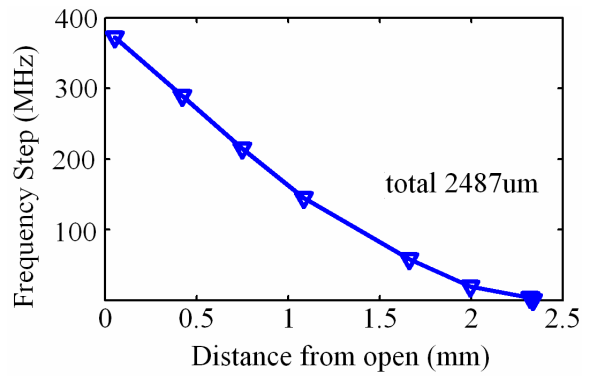

Fig.9. Frequency step vs distance from open end.

transmission line. This means that to realize fine frequency tuning steps, instead of trying to control very small capacitors, medium size capacitors can simply be placed near the shorted end giving effectively fine tuning steps while avoiding being overwhelmed by parasitic capacitance such as that from the switch. Ideally, infinitely small frequency steps can be achieved simply by placing capacitors closer to shorted end, while the practical limit is determined by how accurate the short circuit is, and knowing its exact position. For larger tuning steps, capacitors should be placed near the open end where the capacitance's sensitivity is highest. The general idea of the new resonator is shown in Fig. 7 where $\mathrm{C} 1$ to $\mathrm{CN}$ may represent one capacitor or a capacitor bank.

This paper proposes a new DCO using the capacitance's sensitivity in relation to its position on a shorted transmission line to increase the resolution to the DCO shown in Fig.7. Capacitors $\mathrm{C} 0$ to $\mathrm{C} 6$ are distributed along the transmission line using the same medium size MOS capacitors of 800fF switching step. Capacitor $\mathrm{C} 7$ is placed nearly parallel with $C 6$, however, it utilizes minimum size MOS transistor for $0.18 \mu \mathrm{m}$ CMOS process. The transmission line was made from coplanar strip lines (CSL) and a noise filtering capacitor was placed to filter up converted current source noise [9].

\section{MEASUREMENT}

The experimental circuit was fabricated in $0.18 \mu \mathrm{m}$ CMOS process. Due to the large number of control inputs, the on wafer probes were used only for the internal VCO, output buffer biasing and for measuring the output signal. The control pins were wire bonded manually to a package, the package was placed on a printed circuit board and controlled manually from the outside. 


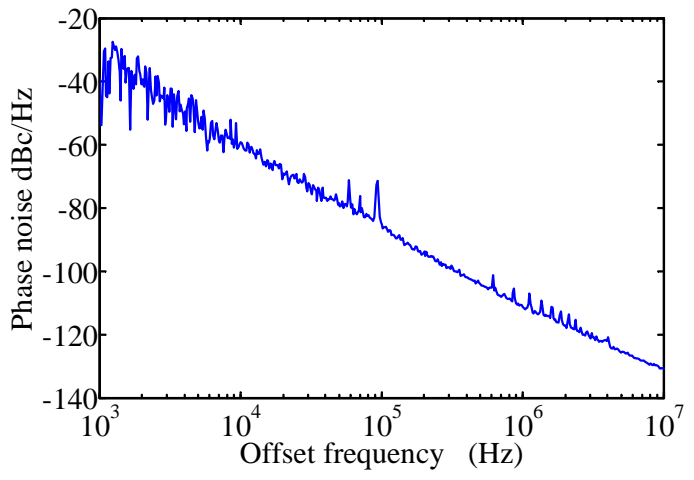

Fig. 10. Measured phase noise.

External amplification and frequency division by 2 before being analyzed by the VCO analyzer was necessary due to the VCO analyzer's limited frequency range.

The capacitors were switched between low and high capacitance one by one and the oscillation frequency was measured. The rescaled measured result (since the frequency was divided by 2 for measurement) is shown in Fig. 8 while the resulting frequency step is shown in Fig.9. As expected, the oscillation frequency changed with the position of the capacitors switched on even though the sizes of the capacitors are the same. The oscillation frequency exhibit a curve inverse to the effective capacitor switching position as shown in Fig.6 as expected. Maximum frequency step using the medium size MOS capacitor placed furthest away from the shorted end was $372.6 \mathrm{MHz}$ while minimum step size for the same medium size MOS capacitor placed near the shorted end $(150 \mu \mathrm{m}$ from short) was $3.45 \mathrm{MHz}$ proving that changing only the position of the capacitor alone could change the frequency tuning sensitivity by more than 100times. The minimum size capacitor placed at approximately the same position as the medium size capacitor near the shorted end showed a measured frequency step of less than $100 \mathrm{kHz}$ at $9 \mathrm{GHz}$. It is possible to increase the frequency step sensitivity and achieve even greater tuning control resolution by placing the capacitors nearer to the shorted end.

The measured phase noise (PN) is shown in Fig. 10. The oscillator's measured phase noise is $-105 \mathrm{dBc} / \mathrm{Hz} @ 1 \mathrm{MHz}$ offset at the oscillation frequency of $9.224 \mathrm{GHz}$ (Measured \%2 PN is $111 \mathrm{dBc} / \mathrm{Hz} @ 1 \mathrm{MHz}$ ). This phase noise is achieved with $5 \mathrm{~mA}$ bias current at the supply voltage of $1.8 \mathrm{~V}$. Phase noise can potentially improved by ground shielding of the CSL and replacing the sensitive varactors with capacitors and switches.

\section{CONCLUSION}

In this paper we have shown how the capacitance's sensitivity to position of the shorted distributed resonator can be used to control the oscillation frequency. A $9 \mathrm{GHz} \mathrm{DCO}$ was designed, and measurement results confirm that the fine frequency tuning can be achieved by varying the capacitor's position on the shorted transmission line. Measured results show

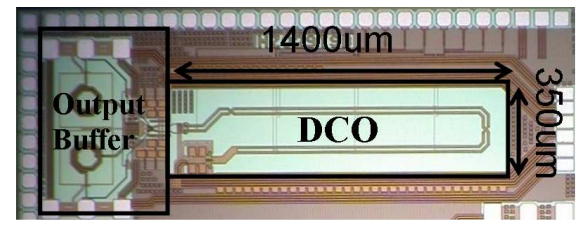

Fig. 11. Chip photo.

that more than 100 times frequency step change can be achieved by varying the position of loading capacitor alone. This enables an extra dimension in design of digital controlled oscillator potentially offering both wide tuning range and fine frequency tuning simultaneously. Due to the potential to reduce the frequency step size in the ADPLL, fine frequency steps for future higher frequency oscillators is possible while the requirements for high speed delta sigma dithering could potentially could be reduced.

\section{ACKNOWLEDGMENT}

The authors would like to thank Masu Laboratory and Masu Lab members for lending of the measurement equipment and support for measurement. Special thanks to Kasuma Ohashi for measurement suggestions, and Yasuhide Kuramochi for suggestions on PCB design for measurement. This work was supported by VLSI Design and Education Center (VDEC), the University of Tokyo in collaboration with Cadence Design Systems, Inc and Agilent Technologies Japan, Ltd.

\section{REFERENCES}

[1] R B. Staszewski, et al. "All-Digital TX Frequency Synthesizer and Discrete-Time Receiver for Bluetooth Radio in 130-nm CMOS,” IEEE J. Solid-State Circuits vol. 39, pp. 2278-2291, December 2004.

[2] R. B. Staszewski, Dirk Leipold and Poras T. Balsara, "Direct Frequency Modulation of an ADPLL for Bluetooth/GSM With Injection Pulling Elimination”, IEEE Trans. Circuits and Systems II. Vol 52, pp. 339-343, June 2005

[3] R. B. Staszewski and Poras T. Balsara "All-Digital PLL With Ultra Fast Settling", IEEE Trans. Circuits and Systems II, vol 54, pp 181-185, February 2007.

[4] W. Chaivipas and A. Matsuzawa, "Analysis and Design of Direct Reference Feed-Forward Compensation for Fast-Settling All-Digital Phase-Locked Loop", IEICE Trans. Electron, vol. 90, pp 793-501, April 2007

[5] W. Chaivipas, A. Matsuzawa, and P. C. Oh., "Feed-Forward compensation technique for all digital phase locked loop based synthesizers," Proc. ISCAS, pp. 3209-3212, May 2006.

[6] C. Hung, R. Staszewski, N. Varton, M. Lee and D. Leipold, “A Digitally Controlled Oscillator System for SAW-Less Transmitters in Cellular Handsets", IEEE J. Solid-State Circuits, vol.. 41, pp 1160-1170, May 2006

[7] R. B. Staszewski, C. Hung, N. Barton, M Lee and D. Leipold, "A Digitally Controlled Oscillator in a $90 \mathrm{~nm}$ Digital CMOS Process for Mobile Phones", IEEE J. Solid-State Circuits, vol. 40, pp 2203-2211, November 2005

[8] D. M. Pozar, Microwave Engineering, $2^{\text {nd }}$ ed., United States of America 1998, pp 154-155

[9] E. Hegazi, H. Sjoland, and A. A. Abidi, "A Filtering Technique to Lower LC Oscillator Phase Noise", IEEE J. Solid-State circuits, vol. 36, pp 19211230, December 2001 